\title{
Erratum to: On the Homology of Completion and Torsion
}

\author{
Marco Porta $^{1} \cdot$ Liran Shaul $^{2} \cdot$ Amnon Yekutieli $^{1}$
}

Published online: 20 August 2015

(C) Springer Science+Business Media Dordrecht 2015

\section{Erratum to: Algebr Represent Theor (2014) 17:31-67 DOI 10.1007/s10468-012-9385-8}

In this note we correct two errors in our paper [2].

(1) There is an error in the proof of Theorem 7.12 (GM Duality) of [2]. The statement itself is correct, and it is repeated as Theorem 9 below, with a correct proof.

(2) Just before formula (3.12) in [2], we said that " $\operatorname{Mod}_{\mathfrak{a} \text {-tor }} A$ is a thick abelian subcategory of Mod $A$ ". This is true if the ideal $\mathfrak{a}$ is finitely generated, but might be false otherwise. There is no implication of this error on the rest of the paper, since WPR ideals are by definition finitely generated. We thank R. Vyas for mentioning this error to us.

In the rest of the note we recall the definitions and results from [2], that are needed to prove Theorem 9.

Let $A$ be a commutative ring, and let $\mathfrak{a}$ be a weakly proregular ideal in it (see [2, Definition 4.21]). We choose a finite sequence $\boldsymbol{a}=\left(a_{1}, \ldots, a_{n}\right)$ that gener-

The online version of the original article can be found at http://dx.doi.org/10.1007/s10468-012-9385-8.

Amnon Yekutieli

amyekut@math.bgu.ac.il

Marco Porta

marcoporta1@libero.it

Liran Shaul

Liran.Shaul@uantwerpen.be

1 Department of Mathematics Ben, Gurion University, Be'er Sheva 84105, Israel

2 Departement Wiskunde-Informatica, Universiteit Antwerpen, Middelheim Campus, Middelheimlaan 1, 2020 Antwerp, Belgium 
ates the ideal $\mathfrak{a}$. The telescope complex associated to this sequence is $\operatorname{Tel}(A ; \boldsymbol{a})$. There is a canonical homomorphism of complexes

$$
u_{\boldsymbol{a}}: \operatorname{Tel}(A ; \boldsymbol{a}) \rightarrow A .
$$

See [2, Section 5]. Let us write $T:=\operatorname{Tel}(A ; \boldsymbol{a})$ and $u:=u_{\boldsymbol{a}}$. For any complex of $A$ modules $M$, we identify $M$ with $A \otimes_{A} M$ and with $\operatorname{Hom}_{A}(A, M)$. This allows us to form the homomorphisms

$$
u \otimes \mathrm{id}: T \otimes_{A} M \rightarrow M
$$

and

$$
\operatorname{Hom}(u, \mathrm{id}): M \rightarrow \operatorname{Hom}_{A}(T, M) .
$$

The mistake in the proof of [2, Theorem 7.12] was as follows. We had claimed that for any K-projective complex $P$, the homomorphism of complexes

$$
\operatorname{Hom}(u, \mathrm{id}): T \otimes_{A} P \rightarrow \operatorname{Hom}_{A}\left(T, T \otimes_{A} P\right)
$$

is a quasi-isomorphism. But this is false. Here is a counterexample:

Example 2 Take $A:=\mathbb{K}[[t]]$, the ring of formal power series over a field $\mathbb{K}$, with $\mathfrak{a}:=(t)$. Consider the complex $P:=A$. Then there are derived category isomorphisms

$$
T \otimes_{A} P \cong T \cong \mathrm{R} \Gamma_{\mathfrak{a}}(A) \cong \mathrm{H}^{1}\left(\mathrm{R} \Gamma_{\mathfrak{a}}(A)\right)[-1]
$$

and

$$
\operatorname{Hom}_{A}\left(T, T \otimes_{A} P\right) \cong \operatorname{Hom}_{A}(T, T) \cong \mathrm{L} \Lambda_{\mathfrak{a}}(A) \cong \Lambda_{\mathfrak{a}}(A) \cong A
$$

The next two results from [2] will be crucial for the proof of Theorem 9, so we copy them.

Proposition 3 [2, Proposition 5.8] Let A be a commutative ring, let $\mathfrak{a}$ be a weakly proregular ideal in $A$, and let $\boldsymbol{a}$ be a finite sequence that generates the ideal $\mathfrak{a}$. For any $M \in$ $\mathrm{D}(\operatorname{Mod} A)$ there is an isomorphism

$$
v_{\boldsymbol{a}, M}^{\mathrm{R}}: \mathrm{R} \Gamma_{\mathfrak{a}}(M) \stackrel{\simeq}{\rightarrow} \operatorname{Tel}(A ; \boldsymbol{a}) \otimes_{A} M
$$

in $\mathrm{D}(\operatorname{Mod} A)$. The isomorphism $v_{\boldsymbol{a}, M}^{\mathrm{R}}$ is functorial in $M$, and the diagram

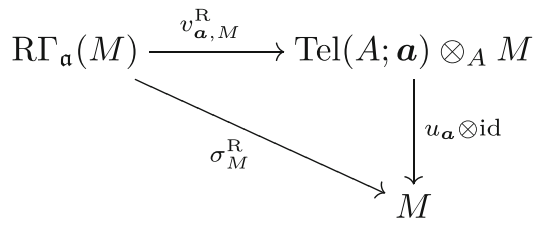

in $\mathrm{D}(\operatorname{Mod} A)$ is commutative.

Proposition 4 [2, Corollary 5.25] Let A be a commutative ring, let $\mathfrak{a}$ be a weakly proregular ideal in $A$, and let $\boldsymbol{a}$ be a finite sequence that generates the ideal $\mathfrak{a}$. For any $M \in \mathrm{D}(\operatorname{Mod} A)$ there is an isomorphism

$$
\operatorname{tel}_{\boldsymbol{a}, M}^{\mathrm{L}}: \operatorname{Hom}_{A}(\operatorname{Tel}(A ; \boldsymbol{a}), M) \rightarrow \operatorname{L} \Lambda_{\mathfrak{a}}(M)
$$


in $\mathrm{D}(\operatorname{Mod} A)$. The isomorphism tel $_{\boldsymbol{a}, M}^{\mathrm{L}}$ is functorial in $M$, and the diagram

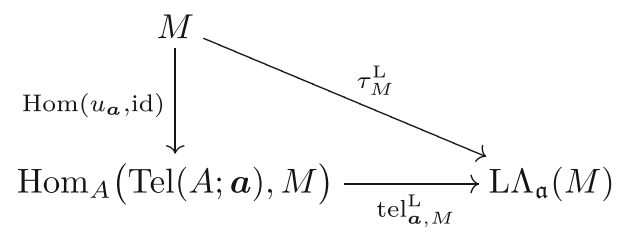

in $\mathrm{D}(\operatorname{Mod} A)$ is commutative.

Here are four lemmas that will be needed for the proof of Theorem 9. We retain the assumptions of the propositions above, and the shorthand $T:=\operatorname{Tel}(A ; \boldsymbol{a})$ and $u:=u_{\boldsymbol{a}}$. The next lemma replaces the problematic (1).

Lemma 5 For any complex of A-modules $M$, the homomorphism of complexes

$$
\operatorname{Hom}_{A}(\mathrm{id}, u \otimes \mathrm{id}): \operatorname{Hom}_{A}\left(T, T \otimes_{A} M\right) \rightarrow \operatorname{Hom}_{A}(T, M)
$$

is a quasi-isomorphism.

Proof We will prove that this is an isomorphism in $\mathrm{D}(\operatorname{Mod} A)$. Using Propositions 3 and 4 , we may replace the given morphism with

$$
\mathrm{L} \Lambda_{\mathfrak{a}}\left(\sigma_{M}^{\mathrm{R}}\right): \mathrm{L} \Lambda_{\mathfrak{a}}\left(\mathrm{R} \Gamma_{\mathfrak{a}}(M)\right) \rightarrow \mathrm{L} \Lambda_{\mathfrak{a}}(M) .
$$

According to [2, Lemma 7.2], the morphism $\mathrm{L} \Lambda_{\mathfrak{a}}\left(\sigma_{M}^{\mathrm{R}}\right)$ is an isomorphism.

The following lemma is actually stated correctly in the proof of [2, Theorem 7.12], but we repeat it for completeness.

Lemma 6 For any complex of A-modules $M$, the homomorphism of complexes

$$
\mathrm{id} \otimes \operatorname{Hom}(u, \mathrm{id}): T \otimes_{A} M \rightarrow T \otimes_{A} \operatorname{Hom}_{A}(T, M)
$$

is a quasi-isomorphism.

Proof As in the proof of Lemma 5, it suffices to prove that the morphism

$$
\mathrm{R} \Gamma_{\mathfrak{a}}\left(\tau_{M}^{\mathrm{L}}\right): \mathrm{R} \Gamma_{\mathfrak{a}}(M) \rightarrow \mathrm{R} \Gamma_{\mathfrak{a}}\left(\mathrm{L} \Lambda_{\mathfrak{a}}(M)\right)
$$

in $\mathrm{D}(\operatorname{Mod} A)$ is an isomorphism. This is true by [2, Lemma 7.6].

Lemma 7 For any complex of A-modules $N$, the homomorphism of complexes

$$
\operatorname{Hom}\left(\operatorname{id}_{T}, \operatorname{Hom}\left(u, \operatorname{id}_{N}\right)\right): \operatorname{Hom}_{A}(T, N) \rightarrow \operatorname{Hom}_{A}\left(T, \operatorname{Hom}_{A}(T, N)\right)
$$

is a quasi-isomorphism.

Proof Using Hom-tensor adjunction, we can replace the given homomorphism with the homomorphism

$$
\operatorname{Hom}(u \otimes \mathrm{id}, \mathrm{id}): \operatorname{Hom}_{A}(T, N) \rightarrow \operatorname{Hom}_{A}\left(T \otimes_{A} T, N\right)
$$

in $\mathrm{C}(\operatorname{Mod} A)$, that is isomorphic to it. Now, by [2, Corollary 7.9], the homomorphism

$$
u \otimes \mathrm{id}_{T}: T \otimes_{A} T \rightarrow T
$$


is a homotopy equivalence. Hence $\operatorname{Hom}\left(u \otimes \mathrm{id}_{T}, \mathrm{id}_{N}\right)$ is a quasi-isomorphism.

Lemma 8 For any complex of A-modules $N$, the homomorphism of complexes

$$
u \otimes \operatorname{id}_{T} \otimes \operatorname{id}_{N}: T \otimes_{A} T \otimes_{A} N \rightarrow T \otimes_{A} N
$$

is a quasi-isomorphism.

Proof We already know that $u \otimes \mathrm{id}_{T}$ is a homotopy equivalence.

Theorem 9 (GM Duality) Let A be a commutative ring, and let $\mathfrak{a}$ be a weakly proregular ideal in $A$. For any $M, N \in \mathrm{D}(\operatorname{Mod} A)$ the morphisms

$$
\begin{aligned}
\operatorname{RHom}_{A}\left(\mathrm{R} \Gamma_{\mathfrak{a}}(M), \mathrm{R} \Gamma_{\mathfrak{a}}(N)\right) & \stackrel{\operatorname{RHom}\left(\mathrm{id}, \sigma_{N}^{\mathrm{R}}\right)}{\longrightarrow} \operatorname{RHom}_{A}\left(\mathrm{R} \Gamma_{\mathfrak{a}}(M), N\right) \\
& \stackrel{\operatorname{RHom}\left(\mathrm{id}, \tau_{N}^{\mathrm{L}}\right)}{\longrightarrow} \operatorname{RHom}_{A}\left(\mathrm{R} \Gamma_{\mathfrak{a}}(M), \mathrm{L} \Lambda_{\mathfrak{a}}(N)\right) \\
& \stackrel{\mathrm{RHom}\left(\sigma_{M}^{\mathrm{R}}, \mathrm{id}\right)}{\longleftarrow} \operatorname{RHom}_{A}\left(M, \operatorname{L} \Lambda_{\mathfrak{a}}(N)\right) \\
& \stackrel{\operatorname{RHom}\left(\tau_{M}^{\mathrm{L}}, \mathrm{id}\right)}{\longleftarrow} \operatorname{RHom}_{A}\left(\operatorname{L} \Lambda_{\mathfrak{a}}(M), \mathrm{L} \Lambda_{\mathfrak{a}}(N)\right)
\end{aligned}
$$

in $\mathrm{D}(\operatorname{Mod} A)$ are isomorphisms.

Proof Choose a weakly proregular sequence $\boldsymbol{a}$ that generates $\mathfrak{a}$, and write $T:=\operatorname{Tel}(A ; \boldsymbol{a})$ and $u:=u_{\boldsymbol{a}}$. Next choose a K-projective resolution $P \rightarrow M$, and a K-injective resolution $N \rightarrow I$. The complex $T \otimes_{A} P$ is K-projective, and the complex $\operatorname{Hom}_{A}(T, I)$ is K-injective.

By Propositions 3 and 4, we can replace the diagram above with the diagram

$$
\begin{aligned}
\operatorname{Hom}_{A}\left(T \otimes_{A} P, T \otimes_{A} I\right) & \\
& \stackrel{\operatorname{Hom}(\mathrm{id} \otimes \mathrm{id}, u \otimes \mathrm{id})}{\longrightarrow} \operatorname{Hom}_{A}\left(T \otimes_{A} P, I\right) \\
& \stackrel{\operatorname{Hom}(\mathrm{id} \otimes \mathrm{id}, \operatorname{Hom}(u, \mathrm{id}))}{\longrightarrow} \operatorname{Hom}_{A}\left(T \otimes_{A} P, \operatorname{Hom}_{A}(T, I)\right) \\
& \stackrel{\operatorname{Hom}(u \otimes \mathrm{id}, \operatorname{Hom}(\mathrm{id}, \mathrm{id}))}{\longleftarrow} \operatorname{Hom}_{A}\left(P, \operatorname{Hom}_{A}(T, I)\right) \\
& \stackrel{\operatorname{Hom}(\operatorname{Hom}(\mathrm{id}, u), \operatorname{Hom}(\mathrm{id}, \mathrm{id}))}{\longleftarrow} \operatorname{Hom}_{A}\left(\operatorname{Hom}_{A}(T, P), \operatorname{Hom}_{A}(T, I)\right)
\end{aligned}
$$

in $\mathrm{C}(\operatorname{Mod} A)$. We will prove that all these homomorphisms are quasi-isomorphisms.

First we deal with the two forward facing homomorphisms in (10). By Hom-tensor adjunction we can move the complex $P$ to the left in all expressions, thus obtaining an isomorphic diagram

$$
\begin{aligned}
& \operatorname{Hom}_{A}\left(P, \operatorname{Hom}_{A}\left(T, T \otimes_{A} I\right)\right) \\
& \quad \stackrel{\operatorname{Hom}(\mathrm{id}, \operatorname{Hom}(\mathrm{id}, u \otimes \mathrm{id}))}{\longrightarrow} \operatorname{Hom}_{A}\left(P, \operatorname{Hom}_{A}(T, I)\right) \\
& \stackrel{\operatorname{Hom}(\mathrm{id}, \operatorname{Hom}(\mathrm{id}, \operatorname{Hom}(u, \mathrm{id})))}{\longrightarrow} \operatorname{Hom}_{A}\left(P, \operatorname{Hom}_{A}\left(T, \operatorname{Hom}_{A}(T, I)\right)\right)
\end{aligned}
$$

in $\mathrm{C}(\operatorname{Mod} A)$. Because $P$ is $\mathrm{K}$-projective, it suffices to prove that

$$
\begin{aligned}
& \operatorname{Hom}_{A}\left(T, T \otimes_{A} I\right) \\
& \quad \stackrel{\operatorname{Hom}(\mathrm{id}, u \otimes \mathrm{id})}{\longrightarrow} \operatorname{Hom}_{A}(T, I) \\
& \stackrel{\operatorname{Hom}(\mathrm{id}, \operatorname{Hom}(u, \mathrm{id}))}{\longrightarrow} \operatorname{Hom}_{A}\left(T, \operatorname{Hom}_{A}(T, I)\right)
\end{aligned}
$$


are quasi-isomorphisms. This is true by Lemmas 5 and 7.

Now we deal with the backward facing homomorphisms in (10). Using Hom-tensor adjunction, we can move the rightmost occurrence of the complex $T$ to the left, thus obtaining an isomorphic diagram

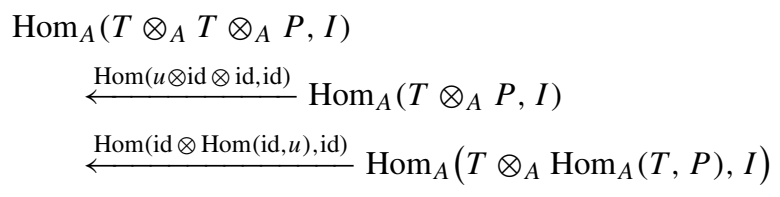

in $\mathrm{C}(\operatorname{Mod} A)$. To prove these are quasi-isomorphisms, we can remove the $\mathrm{K}$-injective complex $I$. Thus it suffices to show that

$$
\begin{aligned}
T \otimes_{A} T \otimes_{A} P \\
\stackrel{u \otimes \mathrm{id} \otimes \mathrm{id}}{\longrightarrow} T \otimes_{A} P \\
\stackrel{\mathrm{id} \otimes \operatorname{Hom}(u, \mathrm{id})}{\longrightarrow} T \otimes_{A} \operatorname{Hom}_{A}(T, P)
\end{aligned}
$$

are quasi-isomorphisms. This is true by Lemmas 6 and 8 .

We should remark that there was a correct proof of a weaker version of Theorem 9 in earlier versions of the paper [2] (the assumption was that $A$ is noetherian). This weaker statement had already been proved in [1].

\section{References}

1. Alonso, L., Jeremias, A., Lipman, J.: Local homology and cohomology on schemes. Ann. Sci. ENS 30, 1-39 (1997). Correction, availabe online at http://www.math.purdue.edu/ lipman/papers/ homologyfix.pdf

2. Porta, M., Shaul, L., Yekutieli, A.: On the homology of completion and torsion. Algebras and Repesentation Theory 17, 31-67 (2014) 